\title{
Structural dependence of CsI(TI) film scintillation properties
}

\author{
A. Ananenko, A. Fedorov, A. Lebedinsky, P. Mateychenko, V. Tarasov, Yu. Vidaj \\ Institute for Scintillation Materials, NAS of Ukraine, 60, Lenin str., 61001 Kharkov, Ukraine \\ Phone: +380 (572) 307958 ; fax: +380 (572) 3210 82; E-mail: fedorov@xray.isc.kharkov.com
}

\begin{abstract}
Scintillating CsI(Tl) films were obtained by vacuum deposition on single crystalline LiF substrates and non-orienting glass substrates. Their structure and morphology were examined by X-ray diffraction and scanning electron microscopy. Scintillation properties of films dependent on their structure are discussed.
\end{abstract}

Keywords: scintillator, vacuum deposition, structure, pulse height spectra.

Paper received 07.06.04; accepted for publication 21.10.04.

\section{Introduction}

Alkali halide crystals, including CsI(Tl), are used widely as scintillation medium for the high energy particles and ionizing radiation detectors. Vacuum deposited $\mathrm{CsI}(\mathrm{Tl})$ films were firstly used as X-ray image intensifiers in electron-optic converters [1]. In the past decade, digital Xray imaging systems were developed using vacuum deposited CsI(Tl) layers coupled with CCD or amorphous silicon detector arrays [2]. The particular feature of CsI(Tl) film is its ability to grow in columnar morphology [3]. This reduces the lateral light spread and promote the directional light passing due to the total internal reflection on column boundaries. Combination of CsI(Tl) film properties affords the high spatial resolution, X-ray stopping power and conversion efficiency. The task of image formation in X-ray radiation at sufficient resolution and low radiation dose is important for medical purposes [4].

Deposition conditions of CsI(Tl) films usually provide the polycrystalline film structure [5] that may hinder mentioned directional light passing due to additional scattering. In this work, the investigations of structural and scintillation characteristics of CsI(Tl) films obtained on orienting and amorphous substrates are presented. Influence of deposition conditions on the films properties is discussed.

\section{Experimental}

CsI(Tl) films were obtained using the physical vapor deposition technique in vacuum better than $5 \cdot 10^{-6}$ Torr from CsI powder with $0.1 \mathrm{wt} . \% \mathrm{Tl}$ contents. The deposition rate was measured using a quartz oscillator placed in the vapour flux and precisely controlled through the source furnace temperature adjustment by computer driven interface.

The film crystalline structure was examined using Xray diffraction in $\mathrm{CuK} \alpha$ radiation. Structure perfectness of single crystalline specimens was estimated by rocking curves obtained on the double crystal X-ray spectrometer with Si monochromator fitted on (400) $\mathrm{CuK} \alpha_{1}$ reflection.

Scanning electron microscopy was used to observe the film morphology.

Scintillation pulse height spectra were obtained using the Hamamatsu 1307 photomultiplier connected to the multichannel amplitude analyser.

\section{Results and discussion}

It is known that the structure of vacuum deposited films is strongly dependent on the growing conditions. The most significant parameters are crystalline state of substrate, misfit value, substrate temperature, growing rate. In this 


\section{A. Ananenko et al.: Structural dependence of CsI(TI) film scintillation properties}

work, CsI(Tl) films were deposited onto the (100) cleavage planes of LiF single crystal used as an orienting substrate and onto the amorphous glass substrates. In both cases columnar morphology of films was observed (Fig. 1). Deposition on LiF substrates was executed at $573 \mathrm{~K}$ substrate temperature and condensation rates from a few $\AA /$ s to about $50 \AA / s$. A comparely high substrate temperature that ensures sufficiently high mobility of adatoms on the surface and low deposition rate that means low supersaturation may provide the epitaxial growth. $\mathrm{X}$-ray diffraction pattern of film grown in such mode presented in Fig. $2 a$ exhibits strong preferred orientation in [110] and [112] crystallographic directions in distinction from [100] substrate orientation. Single crystalline nature of reflecting blocks was proved by X-ray rocking curves. Halfwidths of reflections were from 10 to $24 \mathrm{arc}$ min. for (110) reflections and from 6 to 23 arc min. for (112) reflections of epitaxial films grown at rates below $5 \AA$. Being compared with 2 arc min. halfwidth of the (200) LiF substrate reflection, these values show decent crystalline perfectness of the film. Symmetrical $\theta-2 \theta \mathrm{X}$-ray diffraction allows to define orientation only in the normal direction to the surface. To disclose the complete orientation dependency between the substrate and film,
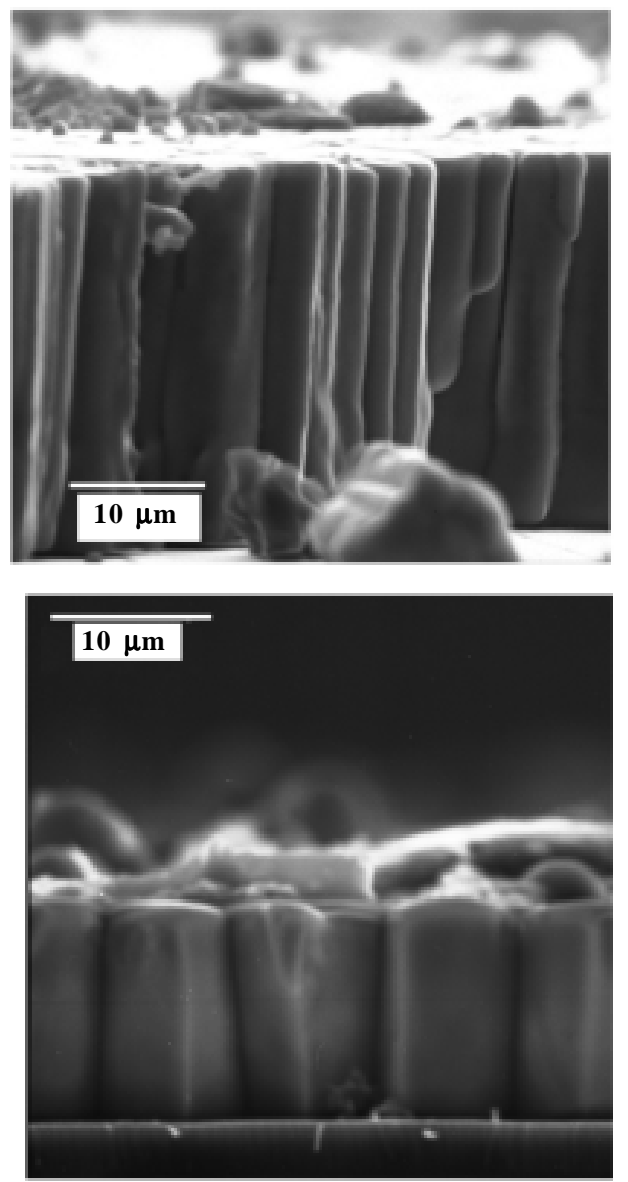

$b$

Fig. 1. SEM images of $\mathrm{CsI}(\mathrm{Tl})$ film cross section on $\mathrm{LiF}(100)$ substrate $(a)$ and glass substrate $(b)$.

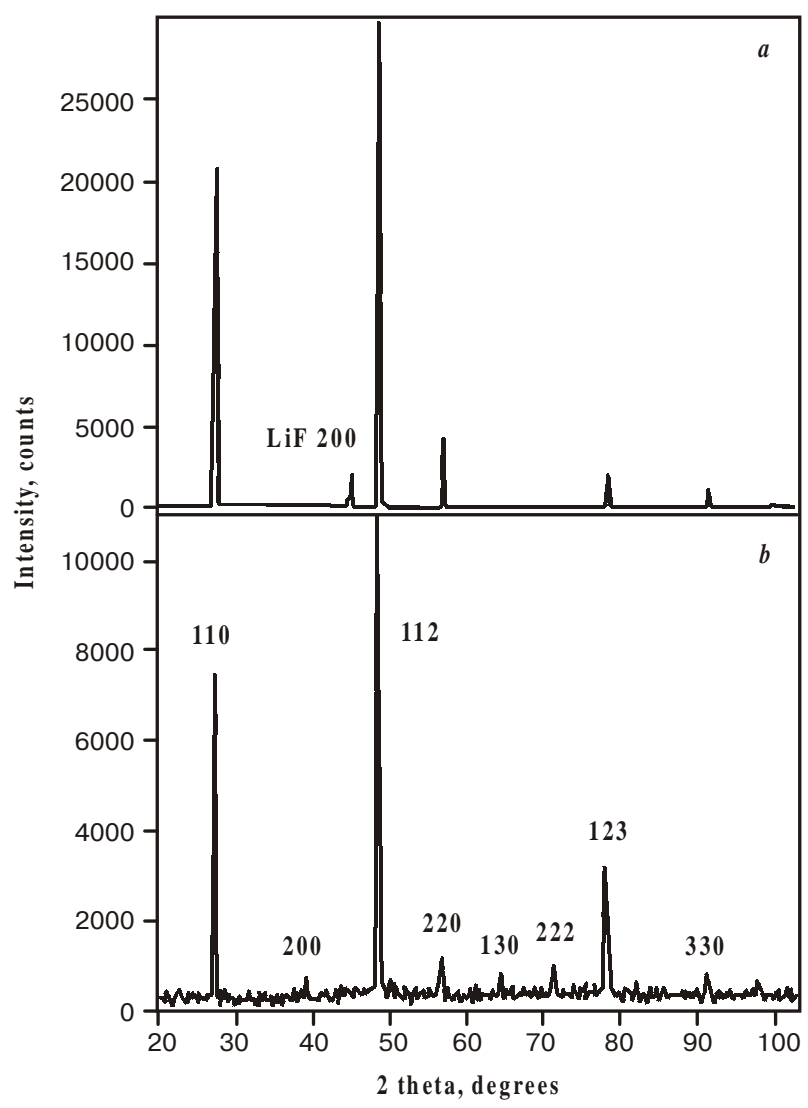

Fig. 2. X-ray diffraction pattern of the CsI(Tl) films on LiF (100) deposited at $573 \mathrm{~K}$ substrate temperature, deposition rate $6 \AA$ A/s (a) and $43 \AA / \mathrm{s}(b)$. CsI reflections indices are denoted in $(b)$.

the relations between reflections from inclined diffraction planes were examined in the asymmetric mode. It was established that lateral orientation of the film crystalline blocks is not arbitrary but entirely defined by the substrate orientation. Corresponding stereographic projections are shown in Fig. 3. The set of found orientations show that columnar crystalline blocks exist in the twinned positions related to one another. These data allow to suppose the structure of substrate-film interface (Fig. 4). It

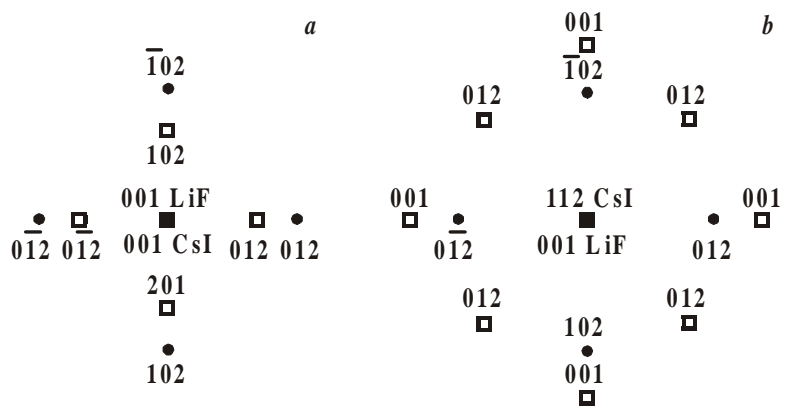

Fig. 3. Strereographic projections of inclined to (100) LiF surface planes. Dark dots correspond to $\mathrm{LiF}$ and light squares to CsI film. CsI blocks orientation (101) (a) and (112) (b).

SQO, 7(3), 2004 


\section{A. Ananenko et al.: Structural dependence of CsI(TI) film scintillation properties}

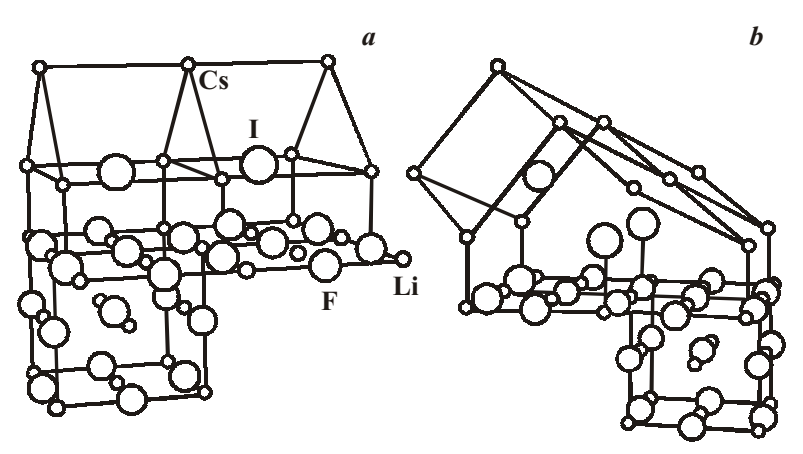

Fig. 4. Model of the interface structure for (110) (a) (112) (b) orientations of the CsI blocks.

follows from this reconstruction that interaction of the substrate and film is very weak due to balanced repulsion and attraction between ions through interface. Taking into account large values of CsI and LiF lattice misfit (4.2\% for [112] orientation and $7.5 \%$ for [110] orientation of blocks) this signifies likely the Volmer-Weber

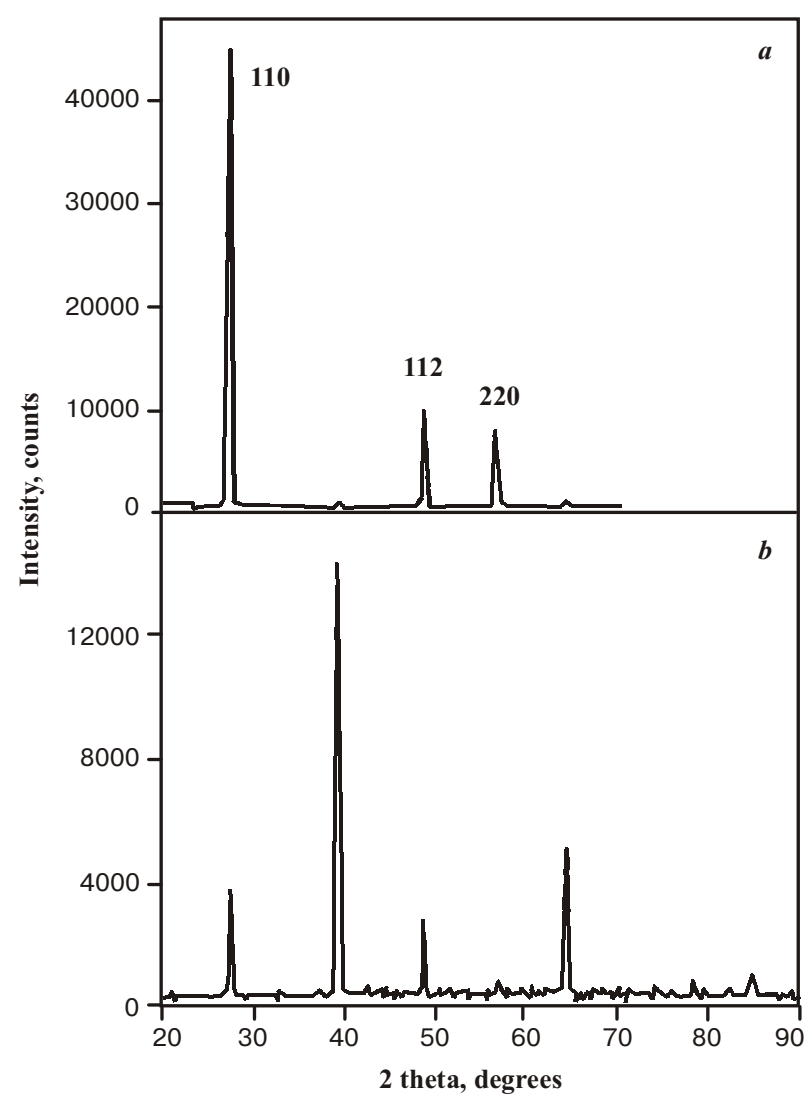

Fig. 5. X-ray diffraction pattern of $\mathrm{CsI}(\mathrm{Tl})$ films on the glass substrate deposited at $573 \mathrm{~K}(a)$ and room $(b)$ substrate temperature.

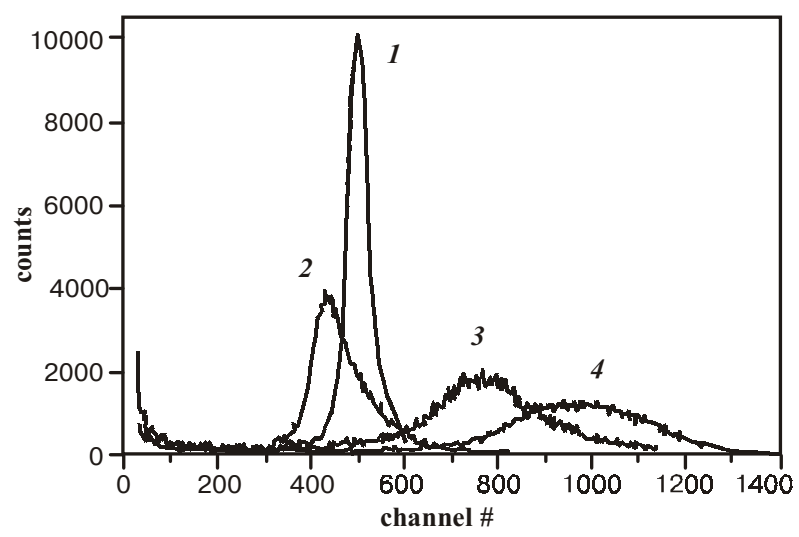

Fig. 6. Pulse height spectra of $\mathrm{CsI}(\mathrm{Tl}) / \mathrm{LiF}$ films $(2-4)$ and standard $\mathrm{CsI}(\mathrm{Tl}) \alpha$-detector (1) obtained using Pu $239 \alpha$-radiation. Film thicknesses equal 5 (2), 10 (3) and $26 \mu \mathrm{m}$ (4). All film spectra are normalized to the $\alpha$-detector peak.

growth mechanism. Increase of the condensation rate to tens $\mathrm{A} / \mathrm{s}$ leads to deterioration of the film crystalline quality. The preferred orientation is retained but the rocking curve halfwidth increases drastically to immeasurable values and the polycrystalline structure emerges as shown in Fig. $2 b$.

Crystalline structure of CsI(Tl) films deposited on the glass substrate is strongly dependent on the substrate temperature. X-ray diffraction patterns in Fig. 5 show that the film deposited at the room temperature possesses the usual polycrystalline CsI structure. An elevated substrate temperature provides appearance of the strong preferred orientations in the deposited layer.

Pulse height spectra of CsI(Tl) films presented in Fig. 6 demonstrate dependence of the amplitude peak position on the film thickness. All fims in this example possess single crystalline structure with good perfectness. The amplitude peak position is defined by the total amount of photons emerged at the counteraction event with a particle, so it expresses the conversion efficiency of this medium. One can notice that the light output of films with more than $10 \mu \mathrm{m}$ thickness is considerably greater than that of the standard $\mathrm{CsI}(\mathrm{Tl}) \alpha$-particle detector used as a reference mark. But this feature was achieved only for single crystalline layers. Reduction of the crystal perfectness of film decreases its light output. Pulse height spectra of the films deposited on the non-orienting glass substrate are shown in Fig. 7. Films were deposited at $573 \mathrm{~K}$ substrate temperature that promotes preferred crystallographic orientation in the film (Fig. 5a), but at different growth rates. The increase of the growth rate leads to the structure deterioration and reduction of the light output. Another difference of the films pulse height spectra is their reduced energy resolution as compared with the standard $\alpha$-detector. Resolution for the $\alpha-$ detector was $10 \%$, and for films it was from 23 to $36 \%$. Most probably, this is the effect of the source depletion of $\mathrm{Tl}$ during evaporation that leads to the non-uniformity of the layer characteristics. 


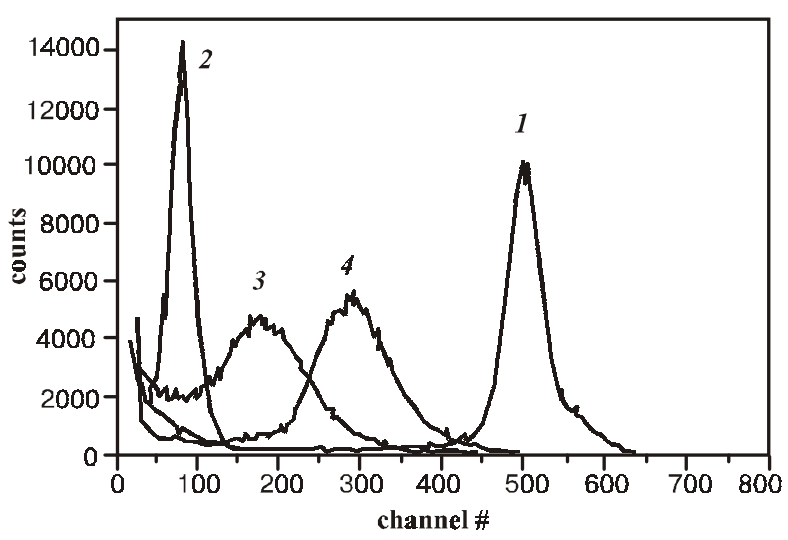

Fig. 7. Pulse height spectra of CsI(Tl)/glass films $(2-4)$ and standard $\mathrm{CsI}(\mathrm{Tl}) \alpha$-detector (1) obtained using Pu $239 \alpha$-radiation. Deposition rates are $12 \AA / s$ (2), $9 \mathrm{~A} / \mathrm{s}$ (3) and $1.3 \AA$ /s (4). All film spectra are normalized to the $\alpha$-detector peak.

\section{Conclusions}

Vacuum deposition of $\mathrm{CsI}(\mathrm{Tl})$ onto the orienting $\mathrm{LiF}$ substrate at appropriate conditions leads to columnar morphology of the grown layer and single crystalline structure of blocks in two orientations, (110) and (112), that are not coincident with (100) orientation of substrate. This may be the result of the great lattice misfit and weak counteraction between the layer and substrate that provides the Volmer-Weber condensation mode and epitaxial growth according to the coincident nodes mechanism. Single crystalline columnar CsI(Tl) films demonstrate an increased scintillation efficiency comparing with the standard CsI(Tl) single crystalline $\alpha$-detector at thicknesses more than $10 \mu \mathrm{m}$. Retreat from the epitaxial growth conditions leads to degradation of the structure perfectness, yet columnar morphology and preferred crystallographic orientation retained. This is accompanied with a decrease of the scintillation light output apparently due to additional light scattering.

\section{References}

1. C.W. Bates, Scintillation Processes in Thin Films of CsI(Na) and $\mathrm{CsI}(\mathrm{Tl})$ used to Low Energy X-rays, Electrons and Protons, in Photo-Electronic Image Devices, Eds. J.D. McGee, D. McMillan, E. Kahan, B.L. Morgan, pp. 451-459, Academic Press, London-New York (1969).

2. H. Wieczorek, G. Fringis, P. Quadflieg, U. Schiebel, T.F.v. Bergen, F.M. Dreesen, M.A.C. Ligtenberg, T. Poorter, CsI:Tl for solid state X-ray detectors, in: Proc. Int. Conf. on Inorganic Scintillators and Their Applications, Eds. P. Dorenbos and C.W.E.van Eijk, pp. 547-554, Delft University Press, Netherlands (1996).

3. T. Jing, G. Cho, J. Drewery, I. Fujieda, S.N. Kaplan, A. Mireshghi, V. Perez-Mendez, D. Wildermuth, Enhanced Columnar Structure in CsI Layer by Substrate Patterning // IEEE Trans. on Nucl. Sci. 39(5), pp. 1195-1198 (1992)

4. S. Hamers, J. Freyschmidt, Digital radiography with an electronic flat-panel detector: First clinical experience in skeletal diagnostics // Medicamundi, 42(3), pp.2-6 (1998).

5. V.V. Nagarkar, T.K. Gupta, S.R. Miller, Y. Klugerman, M.R. Squillante, G. Entine, W.W. Moses, Structured CsI(Tl) Scintillators for X-ray Imaging Applications // IEEE Trans. on Nucl. Sci. 45(3), pp.492-496 (1998). 